\title{
IONIZATION IN ATMOSPHERES OF BROWN DWARFS AND EXTRASOLAR PLANETS. IV. THE EFFECT OF COSMIC RAYS
}

\author{
P. B. Rimmer AND CH. Helling \\ SUPA, School of Physics and Astronomy, University of St. Andrews, St. Andrews KY16 9SS, UK; pr33@st-andrews.ac.uk \\ Received 2013 May 7; accepted 2013 July 10; published 2013 August 21
}

\begin{abstract}
Cosmic rays provide an important source for free electrons in Earth's atmosphere and also in dense interstellar regions where they produce a prevailing background ionization. We utilize a Monte Carlo cosmic ray transport model for particle energies of $10^{6} \mathrm{eV}<E<10^{9} \mathrm{eV}$, and an analytic cosmic ray transport model for particle energies of $10^{9} \mathrm{eV}<E<10^{12} \mathrm{eV}$ in order to investigate the cosmic ray enhancement of free electrons in substellar atmospheres of free-floating objects. The cosmic ray calculations are applied to DRIFT-PHOENIX model atmospheres of an example brown dwarf with effective temperature $T_{\text {eff }}=1500 \mathrm{~K}$, and two example giant gas planets $\left(T_{\text {eff }}=1000 \mathrm{~K}, 1500 \mathrm{~K}\right)$. For the model brown dwarf atmosphere, the electron fraction is enhanced significantly by cosmic rays when the pressure $p_{\text {gas }}<10^{-2}$ bar. Our example giant gas planet atmosphere suggests that the cosmic ray enhancement extends to $10^{-4}-10^{-2}$ bar, depending on the effective temperature. For the model atmosphere of the example giant gas planet considered here $\left(T_{\text {eff }}=1000 \mathrm{~K}\right)$, cosmic rays bring the degree of ionization to $f_{e} \gtrsim 10^{-8}$ when $p_{\text {gas }}<10^{-8}$ bar, suggesting that this part of the atmosphere may behave as a weakly ionized plasma. Although cosmic rays enhance the degree of ionization by over three orders of magnitude in the upper atmosphere, the effect is not likely to be significant enough for sustained coupling of the magnetic field to the gas.
\end{abstract}

Key words: astroparticle physics - brown dwarfs - magnetic reconnection - planets and satellites: atmospheres stars: atmospheres

Online-only material: color figures

\section{INTRODUCTION}

M dwarfs of spectral class M7 and hotter have been discovered to emit quiescent $\mathrm{X}$-rays, and lower mass brown dwarfs emit $\mathrm{X}$-rays intermittently (Berger et al. 2010). Currently, there is no satisfactory explanation for this phenomenon, but there are some promising models that may account for the observed X-ray emissions. For example, Helling et al. (2011a, 2011b) propose that charge build-up on grains may lead to atmospheric ionization to a degree sufficient to couple the magnetic field to a partially ionized atmospheric gas. The magnetic fields would follow the convective atmospheric dynamics, and would become tangled. X-rays would then be the consequence of magnetic reconnection events. This scenario requires a charge density at least $10^{6}$ times greater than predicted by thermal ionization in current model atmospheres (see Helling et al. 2011b, their Figure 2). Other ionizing mechanisms need therefore to be considered. In this paper, we provide a first study of how significant cosmic ray ionization is in the atmospheres of brown dwarfs and giant gas planets.

If cosmic rays contribute significantly to atmospheric ionization in extrasolar planets and brown dwarfs, the effects of this ionization may provide an opportunity to better constrain the energy spectrum of galactic cosmic rays in a variety of planetary atmospheres. A large number of cosmic rays are blocked from Earth by the solar wind (Jokipii 1976), although the Voyager probe is expected to measures the interstellar cosmic ray flux (Webber \& Higbie 2009). Free-floating planets and stellar objects would not be so protected, and the observable effects of cosmic ray ionization in the atmospheres of these objects may provide us an indirect way to determine the spectrum of extrasolar cosmic rays.

Cosmic rays were first discovered because of their ionizing effect on Earth's atmosphere (Hess 1912). Cosmic ray ionization may affect terrestrial climate conditions by enhancing aerosol formation (Pudovkin \& Veretenenko 1995; Shumilov et al. 1996) and initiating discharge events (Ermakov \& Stozhkov 2003; Stozhkov 2003). Grießmeier et al. (2005) investigate the possible impact of cosmic ray showers on biological organisms in extrasolar Earth-like planets with weak magnetic fields by considering the effect of a weaker planetary magnetic field on cosmic ray propagation through the planetary atmosphere. The effect of Earth's electric field on cosmic ray propagation is also being explored. For example, Muraki et al. (2004) found measurable enhancement in cosmic ray intensity when a negative electric field of magnitude $>10 \mathrm{kV} \mathrm{m}^{-1}$ is present in the atmosphere. Bazilevskaya et al. (2008) provide a comprehensive review of the research into connections between cosmic rays and the atmosphere. The effect of cosmic rays on the exospheres of free-floating extrasolar planets and brown dwarfs that form dust clouds in the low atmosphere has not been explored so far. The impact of cosmic rays on the exosphere is of particular interest as it links the underlying atmosphere with the object's magnetosphere and may also help to understand coronal effects in the substellar mass regime.

The cosmic ray opacity of brown dwarf and hot Jupiter atmospheres has been explored by Helling et al. (2012), where the cosmic ray flux is considered to decrease exponentially with the column density of the gas (Umebayashi \& Nakano 1981). The effect of cosmic ray propagation on the rate of ionization of the gas has been modeled in some detail for diffuse conditions in the interstellar medium (Padovani et al. 2009; Rimmer et al. 2012), dense environments (Umebayashi \& Nakano 1981) and the terrestrial atmosphere (Velinov et al. 2009). Rimmer et al. (2012) and Velinov et al. (2009) both employ Monte Carlo models for cosmic ray propagation. Velinov et al. (2009) consider pair-production and particle decay (full Monte Carlo simulation of an atmospheric cascade), and Rimmer et al. (2012) 
consider the energy loss due to ionizing collisions and the effect of a weak magnetic field $(B<1 \mathrm{mG})$ on the cosmic ray spectrum. The models of Velinov \& Mateev (2008) and Velinov et al. (2009) have been tested against various atmospheric profiles and model assumptions (Mishev \& Velinov 2008, 2010). Padovani et al. (2009) numerically solve the propagation integral from Cravens \& Dalgarno (1978), and account for energy lost due to ionization and excitation. Umebayashi \& Nakano (1981) solve a set of Boltzmann transport equations for the cosmic rays, with a term for energy loss due to pair-production of electrons.

In this paper, we explore the impact of cosmic ray ionization on the electron fraction in model atmospheres of an example brown dwarf and two example giant gas planets. To this end, we utilize model atmospheres which do not necessarily resample any known free-floating planets. In order to separate the effect of cosmic rays from external UV photons, we only consider free-floating objects not in proximity to a strong UV field. We utilize one-dimensional (1D) Monte Carlo and analytic cosmic ray propagation methods over a wide range of densities to allow a principle investigation of the effect of cosmic rays.

In order to explore cosmic ray ionization in the atmosphere, propagation of the cosmic rays through both the exosphere and atmosphere must be treated. A simple density profile for the gas in the exosphere is calculated in Section 2, using the Boltzmann transport equation and assuming a Maxwellian distribution for the gas. This is necessary because the exosphere is very likely no longer a collisionally dominated gas, hence the continuum assumption for applying hydrodynamic concepts breaks down (see, e.g., Chamberlain \& Hunten 1987).

We are using DRIFT-PHOENIX model atmosphere structures which are the result of the solution of the coupled equations of radiative transfer, convective energy transport (modeled by mixing length theory), chemical equilibrium (modeled by laws of mass action), hydrostatic equilibrium, and dust cloud formation (Dehn 2007; Helling et al. 2011b; Witte et al. 2009). The dust cloud formation model includes a model for seed formation (nucleation), surface growth and evaporation of mixed materials, and gravitational settling (Helling et al. 2008). The results of the DRIFT-PHOENIX model atmosphere simulations include the gas temperature-gas pressure structure $\left(T_{\text {gas }}, p_{\text {gas }}\right)$, the local gas-phase composition in thermochemical equilibrium, the local electron number densities $\left(n_{\mathrm{e}}\right)$, and the number of dust grains $\left(n_{\mathrm{d}}\right)$ and their sizes $(a)$ dependent on the height in the atmosphere. These models are determined by the effective temperature $T_{\text {eff }}$, the surface gravity $\log (g)$, and the initial elemental abundances. The elemental abundances are set to the solar values throughout this paper.

We consider a model atmosphere of a brown dwarf, with a surface gravity of $\log g=5$ and an effective temperature of $T_{\text {eff }}=1500 \mathrm{~K}$, as well as model atmospheres for two example giant gas planets, both with $\log g=3$. The effective temperatures of free-floating exoplanets are not constrained, allowing us to freely explore this part of the parameter space. We chose the values of $T_{\text {eff }}=1500 \mathrm{~K}$ to allow for direct comparison with earlier work (Helling et al. 2011b) and $T_{\text {eff }}=1000 \mathrm{~K}$, which is the inferred effective temperature of HR $8799 \mathrm{c}$, a giant gas planet $\sim 40 \mathrm{AU}$ from its host star (Marois et al. 2008). The distance of $40 \mathrm{AU}$ is far enough to possibly allow the planet's parameters to be effectively the same as those of a free-floating giant gas planet.

Section 3 describes our cosmic ray transport calculations for cosmic rays of energy $10^{6} \mathrm{eV}<E<10^{12} \mathrm{eV}$. We then calculate the steady-state degree of ionization by cosmic rays. We combine the cosmic ray ionization to the thermal degree of ionization from DRIFT-PHOENIX. Section 4 contains the resulting degree of ionization and includes the effect on the coupling of the magnetic field of the gas.

\section{DENSITY PROFILE OF THE EXOSPHERE}

For modeling purposes, we divide the gas around the planet or brown dwarf into three regions (inward $\rightarrow$ outward): the cloud layer $^{1}$ (lowest), the dust-free upper atmosphere (middle) and the exosphere (highest). The location and extent of the cloud layer is determined by the dust formation and atmosphere model DRIFTPhoenix (Dehn 2007; Helling et al. 2008; Witte et al. 2009). The exosphere is considered here to be the regime where the gas can no longer be accurately modeled as a fluid. This occurs when the mean free path of the gas particles is of the order of the atmospheric scale height. The pressure at which this is the case is the lowest pressure considered in the DRIFT-PHOENIX model atmospheres, and we place the exobase at that height.

Figure 1 provides gas density and cloud particle number density profiles of the three example model atmospheres and exospheres considered here, as well as the mean grain sizes of cloud particles for the model atmosphere of two example giant gas planets $\left(\log g=3, T_{\text {eff }}=1000 \mathrm{~K}, 1500 \mathrm{~K}\right)$ and a brown dwarf $\left(\log g=5, T_{\text {eff }}=1500 \mathrm{~K}\right)$. This grain size profile demonstrates the location of the cloud with respect to the atmospheric temperature and gas density. Figure 2 shows the $(p, T)$ profiles for the model atmospheres.

In order to model cosmic ray transport into a planet's atmosphere, it is necessary to treat all material between the atmosphere and the source of the incident (galactic) cosmic ray spectrum. Since galactic cosmic ray propagation models (Strong \& Moskalenko 1998) and observations of chemical tracers (Indriolo et al. 2007) currently suggest that cosmic rays of energies $10^{6} \mathrm{eV} \lesssim E \lesssim 10^{12} \mathrm{eV}$ are ubiquitous in the diffuse interstellar medium, we treat the cosmic ray spectrum to be that of the galactic spectrum at the "upper edge"2 of the exosphere, and initiate cosmic ray transport at that point.

We calculate the density profile of the exosphere by solving the steady-state collisionless Boltzmann transport equation with a gravitational force term:

$$
\frac{\boldsymbol{p}}{m} \cdot \nabla f+m \boldsymbol{g} \cdot \frac{\partial f}{\partial \boldsymbol{p}}=0,
$$

where $\boldsymbol{p}$ is the momentum vector, $m$ is the particle mass, $\boldsymbol{g}=G M / r^{2} \hat{\boldsymbol{r}}$ is the gravitational field at radial displacement $\boldsymbol{r}$ from the center of mass, $G=6.67 \times 10^{-8} \mathrm{~cm}^{3} \mathrm{~g}^{-1} \mathrm{~s}^{-2}$, and $M$ is the mass of the giant gas planet or brown dwarf. $f(\boldsymbol{x}, \boldsymbol{p})$ $\left(\mathrm{cm}^{-6} \mathrm{~g}^{-3} \mathrm{~s}^{3}\right)$ is the distribution function, representing the number of particles in the volume element $d^{3} x d^{3} p$ at location $(\boldsymbol{x}, \boldsymbol{p})$ in the phase space. We treat the exosphere 1D for the sake of simplicity. Denoting $\left(r, p_{r}\right)$ as the coordinates of interest,

\footnotetext{
1 The cloud layer is part of the atmosphere, because the cloud particles form from the atmospheric gas. Figure 1 indicates the location and extent of the cloud layer.

2 Technically, there is no definitive upper edge to the exosphere. The gas density decreases monotonically, but there is no definitive transition past the exobase. The location of an "upper edge" is therefore somewhat arbitrary. We treat the "upper edge" of the exosphere to be an infinite distance from the model planet or brown dwarf.
} 

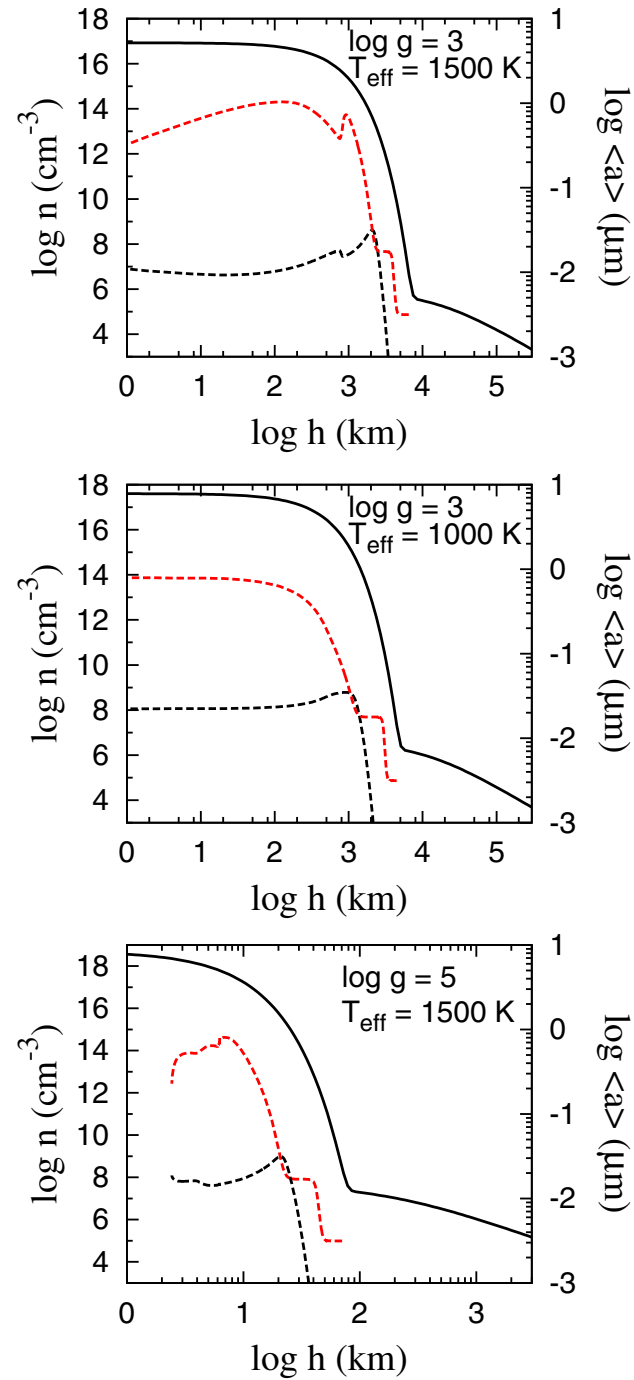

Figure 1. Total number density of the exospheric and atmospheric gas, $n_{\mathrm{gas}}$ $\left(\mathrm{cm}^{-3}\right.$; solid black line, left axis) and of the cloud particles, $n_{\text {dust }}\left(\mathrm{cm}^{-3}\right.$; dashed black line, left axis), and the mean grain radius, $\langle a\rangle$ ( $\mu \mathrm{m}$; dashed red line, right axis), vs. atmospheric height, $h(\mathrm{~km})$, for $\log g=3, T_{\text {eff }}=1500 \mathrm{~K}$ (top), $\log g=3, T_{\text {eff }}=1000 \mathrm{~K}$ (middle) and $\log g=5, T_{\text {eff }}=1500 \mathrm{~K}$ (bottom). The plots show that the cloud layers extend to considerably lower pressures in giant gas planets than in brown dwarfs.

(A color version of this figure is available in the online journal.)

$g=G M / r^{2}$, and Equation (1) becomes:

$$
\frac{p_{r}}{m} \frac{d f\left(r, p_{r}\right)}{d r}-\frac{G m M}{r^{2}} \frac{d f\left(r, p_{r}\right)}{d p_{r}}=0 .
$$

Now $f\left(r, p_{r}\right)\left(\mathrm{cm}^{-4} \mathrm{~g}^{-1} \mathrm{~s}\right)$ is a 1D distribution function representing the number of particles located within a volume element $d^{3} x$ at $r$, with momentum between $p_{r}$ and $p_{r}+d p_{r}$. Both Maxwell-Boltzmann and Lorentzian distribution functions have been applied to the exosphere (Pierrard \& Lemaire 1996). The Maxwell-Boltzmann distribution has been applied to interstellar conditions of similar temperature and density to exosphere conditions, and the calculated deviations from this distribution in the interstellar environment is generally found to be on the order of 1\% (Spitzer 1978, his Section 2.3). We therefore choose the Maxwell-Boltzmann distribution function for our exosphere model. The Maxwell-Boltzmann distribution

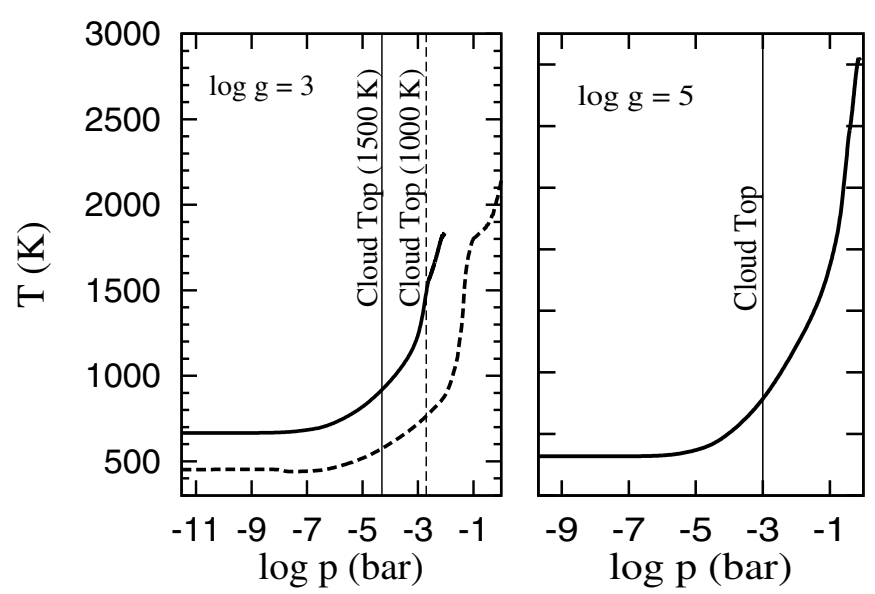

Figure 2. Pressure-temperature profiles for model atmospheres of three example planets, with the parameters $\log g=3, T_{\text {eff }}=1500 \mathrm{~K}$ (left, solid), $\log g=3$, $T_{\text {eff }}=1000 \mathrm{~K}$ and $\log g=5, T_{\text {eff }}=1500 \mathrm{~K}$ (right). The vertical line on each plot indicates the cloud top. Pressures less than the cloud top pressure are considered to be within the upper atmosphere.

is:

$$
f\left(r, p_{r}\right)=\frac{n_{\mathrm{gas}}}{\left(2 \pi m k_{B} T\right)^{1 / 2}} e^{-p_{r}^{2} / 2 m k_{B} T},
$$

where $T$ is the temperature of the gas, $k_{B}=1.4 \times 10^{-16} \mathrm{erg}$ $\mathrm{K}^{-1}$ denotes the Boltzmann constant, and $n_{\text {gas }}\left(\mathrm{cm}^{-3}\right)$ is the gas density profile. We apply Equation (3) to Equation (2). We note that $f$ includes $n_{\text {gas }}=n_{\text {gas }}(r)$ and $T=T(r)$. Employing the product rule for differentiation, the first term of Equation (2) can be written as

$$
\begin{aligned}
\frac{p_{r}}{m} \frac{d f\left(r, p_{r}\right)}{d r}= & \frac{p_{r}}{m} f\left(r, p_{r}\right) \frac{1}{n_{\mathrm{gas}}} \frac{d n_{\mathrm{gas}}}{d r}-\frac{p_{r}}{2 m} f\left(r, p_{r}\right) \frac{1}{T} \frac{d T}{d r} \\
& +\frac{p_{r}^{3}}{2 m^{2} k_{B}} f\left(r, p_{r}\right) \frac{1}{T^{2}} \frac{d T}{d r}
\end{aligned}
$$

and the second term as:

$$
-\frac{G m M}{r^{2}} \frac{d f\left(r, p_{r}\right)}{d p_{r}}=\frac{p_{r}}{k_{B} T} \frac{G M}{r^{2}} f\left(r, p_{r}\right) .
$$

We now reconstruct the transport equation:

$$
\begin{aligned}
& \frac{p_{r}}{m} f\left(r, p_{r}\right) \frac{1}{n_{\mathrm{gas}}} \frac{d n_{\mathrm{gas}}}{d r}-\frac{p_{r}}{2 m} f\left(r, p_{r}\right) \frac{1}{T} \frac{d T}{d r} \\
& \quad+\frac{p_{r}^{3}}{2 m^{2} k_{B}} f\left(r, p_{r}\right) \frac{1}{T^{2}} \frac{d T}{d r}+\frac{p_{r}}{k_{B} T} \frac{G M}{r^{2}} f\left(r, p_{r}\right)=0 .
\end{aligned}
$$

We multiply by $m / p_{r}$ and integrate this equation over $p_{r}$. The first two terms in Equation (6) become:

$$
\begin{gathered}
\int_{-\infty}^{\infty} f\left(r, p_{r}\right) \frac{1}{n_{\mathrm{gas}}} \frac{d n_{\mathrm{gas}}}{d r} d p_{r}=\frac{d n_{\mathrm{gas}}}{d r} \\
\int_{-\infty}^{\infty} \frac{1}{2} f\left(r, p_{r}\right) \frac{1}{T} \frac{d T}{d r} d p_{r}=\frac{n_{\mathrm{gas}}}{2} \frac{1}{T} \frac{d T}{d r} ;
\end{gathered}
$$


The third term in Equation (6) becomes:

$$
\begin{aligned}
& \int_{-\infty}^{\infty} \frac{p_{r}^{2}}{2 m k_{B}} f\left(r, p_{r}\right) \frac{1}{T^{2}} \frac{d T}{d r} d p_{r} \\
& =\frac{n_{\mathrm{gas}}}{\sqrt{\pi}\left(2 m k_{B} T\right)^{3 / 2}} \frac{1}{T} \frac{d T}{d r} \int_{-\infty}^{\infty} p_{r}^{2} e^{-p_{r}^{2} / 2 m k_{B} T} d p_{r}, \\
& =\frac{n_{\mathrm{gas}}}{2} \frac{1}{T} \frac{d T}{d r},
\end{aligned}
$$

and the last term becomes:

$$
\int_{-\infty}^{\infty} \frac{1}{k_{B} T} \frac{G m M}{r^{2}} f\left(r, p_{r}\right) d p_{r}=\frac{G m M}{r^{2}} \frac{n_{\mathrm{gas}}}{k_{B} T} .
$$

Applying Equations (8)-(11) to the integral of Equation (6) over $p_{r}$, the Boltzmann transport equation becomes (with explicit $r$ dependence):

$$
\frac{1}{n_{\mathrm{gas}}(r)} \frac{d n_{\mathrm{gas}}(r)}{d r}=-\frac{G m M}{k_{B} r^{2} T(r)} .
$$

This equation is equivalent to the equation for the distribution function for quasi-collisionless exospheres from Chamberlain \& Hunten (1987, their Equation (7.1.16)), with the angular momentum set to zero and temperature as a function of the radial distance. In order to solve this equation, the temperature would have to be determined via radiative transfer. If we were instead to treat the temperature as a constant in the right-hand side of Equation (12), the equation would become identical to the condition for hydrostatic equilibrium, but this condition is not appropriate for the exosphere. The next simplest functional form for the temperature is the result of setting the average thermal energy, $(3 / 2) k_{B} T$, equal to the virial of the system, which assumes that the system as a whole is stable and bounded. The temperature then becomes:

$$
T(r)=\frac{G m M}{3 k_{B} r} .
$$

Applying Equation (13) to Equation (12), we find:

$$
\frac{1}{n_{\mathrm{gas}}(r)} \frac{d n_{\mathrm{gas}}(r)}{d r}=-\frac{3}{r}
$$

with the solution:

$$
n_{\mathrm{gas}}(r)=n_{c}\left(\frac{r_{c}}{r}\right)^{3}
$$

The inner boundary condition, at the height of the exobase (denoted as $r_{c}$ ) is that the number density $n_{\mathrm{gas}}\left(r_{c}\right)$ is the number density at the exobase, $n_{c}$.

For a sanity check, we compare our results to the more detailed models for the Martian exosphere (Galli et al. 2006). Galli et al. (2006) provide an analytic form of the density profile for the Martian exosphere, and compare it to observation. We are not aware of a more recent analytic expression for exospheric densities that is compared to observational results. Taking their value for the exobase, $r_{c}=3.62 \times 10^{8} \mathrm{~cm}(220 \mathrm{~km}$ above the surface of Mars), setting our $r=h+R_{M}$, where $R_{M}=3.40 \times 10^{8} \mathrm{~cm}$ is the radius of Mars, and using the

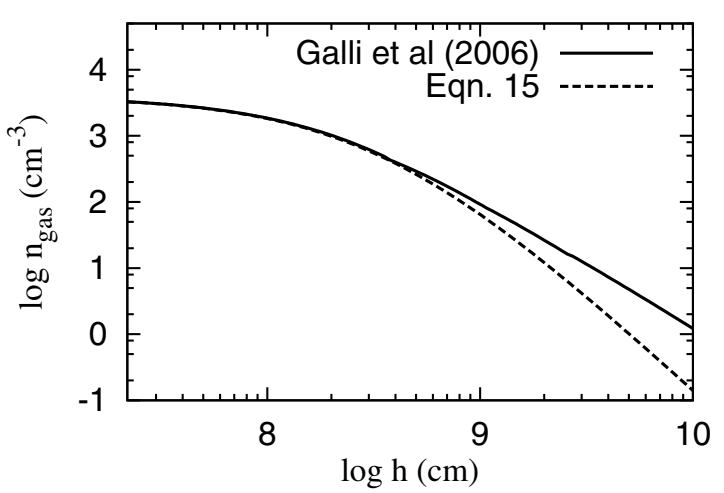

Figure 3. Total gas number density of the Martian exosphere as a function of height above the surface, $h=r-R_{\text {Mars }}$, where $R_{\text {Mars }}=3400 \mathrm{~km}$ is the radius of Mars. The solid line represents an analytical fit to the exospheric model predictions of Galli et al. (2006, their Equation (4)) and the dashed line represents our analytical calculations for the Martian exosphere density, from Equation (15). Our ansatz for the exosphere density profile underestimates the Galli exospheric density profile by about an order of magnitude at $h=10^{10} \mathrm{~cm}$.

density at the exobase from their Equation (4) for our $n_{c}$, we can compare their model results with our own Figure 3. The profile of Galli et al. (2006, their Equation (4)) is within an order of magnitude to our profile for $r<10^{10} \mathrm{~cm}$. Since the two profiles differ significantly only at great distances, when $n \ll n_{c}$, the impact these differences have on the column density is rather small, namely within a factor of two as $r \rightarrow \infty$.

By the use of the virial theorem, we have neglected any thermal emission from the atmosphere. The correct treatment requires a radiative transfer simulation of the atmosphere, which is beyond the scope of this paper. We do not expect these uncertainties to significantly effect cosmic ray transport on free-floating planets and brown dwarfs, for reasons detailed in Section 3. Other relevant uncertainties arise from the neglected planet's rotation, the gravitational pull and radiation pressure from the Sun. Heating from external sources of radiation can also impact the density profile (Hinteregger et al. 1981; Watson et al. 1981; Lammer et al. 2003; Vidal-Madjar et al. 2004; Murray-Clay et al. 2009). Since we are neglecting external sources of UV radiation throughout this paper, the differences arising from radiation pressure and heating will not concern us here. If external UV radiation were to be incorporated (i.e., for young and/or active stars), the impact on exospheric properties could be quite significant. Murray-Clay et al. (2009) found the effect of external photons on the exospheric density profile, not considered in this study, to be up to two orders of magnitude.

Since we consider the exosphere to start at the outermost point in the atmosphere for the DRIFT-PHOENIX model atmosphere under consideration, $n_{0}$ (Equation (15)) is taken to be the outermost density from that model. For the sample brown dwarf atmosphere we consider here $\left(\log (g)=5, T_{\text {eff }}=1500 \mathrm{~K}\right.$, solar metallicity), the exobase density, $n_{c}=3 \times 10^{9} \mathrm{~cm}^{-3}$ which is at $\sim 100 \mathrm{~km}$ above the cloud layer. For the sample giant gas planet $\left(\log (g)=3, T_{\text {eff }}=1500 \mathrm{~K}\right.$, solar metallicity), $n_{c}=4 \times 10^{7} \mathrm{~cm}^{-3}$, which is at $\sim 5000 \mathrm{~km}$ above the cloud layer.

To determine the column density of the exosphere, $N_{\text {exo }}$ $\left(\mathrm{cm}^{-2}\right)$, through which cosmic rays must travel before reaching the upper atmosphere, we perform the integral:

$$
N_{\text {exo }}=\int_{r_{c}}^{\infty} n_{\text {gas }}(r) d r
$$


and applying Equation (15) to Equation (16), we have $N_{\text {exo }} \approx$ $\frac{1}{2} n_{c} r_{c}$. Assuming the radii of both the $\log g=3$ and $\log g=5$ cases to be the radius of Jupiter, $R_{J}=7.1 \times 10^{9} \mathrm{~cm}, N_{\text {exo }} \approx$ $5 \times 10^{18} \mathrm{~cm}^{-2}$ for brown dwarfs and $\approx 7.5 \times 10^{16} \mathrm{~cm}^{-2}$ for a giant gas planet (e.g., Chabrier et al. 2000; Burrows et al. 2001). Although our model is $1 \mathrm{D}$ and neglects rotation and gravitational interaction with other bodies, the cosmic ray transport is not very sensitive to the rather low exospheric column density. Column densities $\lesssim 5 \times 10^{19} \mathrm{~cm}^{-2}$ will not significantly affect cosmic ray transport, according to our model, explained below in Section 3. Errors in the exospheric gas density profile by up to an order of magnitude do not significantly effect our results for cosmic ray transport.

\section{COSMIC RAY TRANSPORT IN THE EXOSPHERE AND ATMOSPHERE}

We have now determined the amount of material a cosmic ray will have to pass through before reaching a certain depth of an atmosphere. In order to determine the average number of cosmic rays to reach a given atmospheric pressure, we must now consider how much energy cosmic rays lose through ionizing collisions, and how many cosmic rays are lost through electron-positron production, pion decay, muon decay, and several other processes. This will allow us to determine both how far into the atmosphere cosmic rays of a given energy reach, and their electron production once they are there. Solving the cosmic ray transport, i.e., the collisional interaction of the individual cosmic ray particles with the surrounding gas, will allow us to estimate an ionization rate for cosmic rays as a function of penetration depth into the atmosphere. The exospheric column density, $N_{\text {exo }}$, will be applied to cosmic ray transport along with the column density between the exobase and a given depth into the atmosphere, $N_{\text {atm }}$, and $N_{\text {col }}=N_{\text {exo }}+N_{\text {atm }}$. In this section, we will determine a cosmic ray ionization rate that depends on the total column, $N_{\text {col }}$.

Cosmic rays of kinetic energy, $E(\mathrm{eV})$, are divided here into low-energy cosmic rays (LECRs, $E<10^{9} \mathrm{eV}$ ) and highenergy cosmic rays (HECRs, $E>10^{9} \mathrm{eV}$ ). LECR transport was calculated by Rimmer et al. (2012) using a Monte Carlo transport model that incorporates the magnetic field effects detailed in Skilling \& Strong (1976) and Cesarsky \& Volk (1978), as well as inelastic collision energy loss. Collisional energy loss is included by first taking the average energy loss per collision, $W(E)(\mathrm{eV})$, from Dalgarno et al. (1999), and the "optical" depth for the cosmic ray. This depth is $\sigma(E) \Delta N$, where $\sigma(E)$ is the total cross-section for inelastic collisions between protons. We use the cross-sections from Rudd et al. (1983) and Padovani et al. (2009).

We apply a Monte Carlo model to determine LECR transport. In this model, detailed in Rimmer et al. (2012), we take 10,000 cosmic rays, and assign each of them two numbers. The first number corresponds to the energy of the individual cosmic ray, and the energies are distributed among the cosmic rays according to the initial cosmic ray flux spectrum (see Figure 4). The second number is a random number of uniform distribution with range $[0,1]$. The cosmic rays are advanced through the atmosphere over a column, $\Delta N$. If the random number is less than $\sigma(E) \Delta N$, the cosmic ray collides with an atmospheric particle, and loses an amount of energy $W(E)$. We choose the size $\Delta N$ so that $\sigma_{\max } \Delta N<1$, where $\sigma_{\max }$ is the maximum cross-section for an inelastic collision between a proton and a hydrogen atom, $\approx 5 \times 10^{-16} \mathrm{~cm}^{2}$ (see Padovani et al. 2009, their Equations (5)
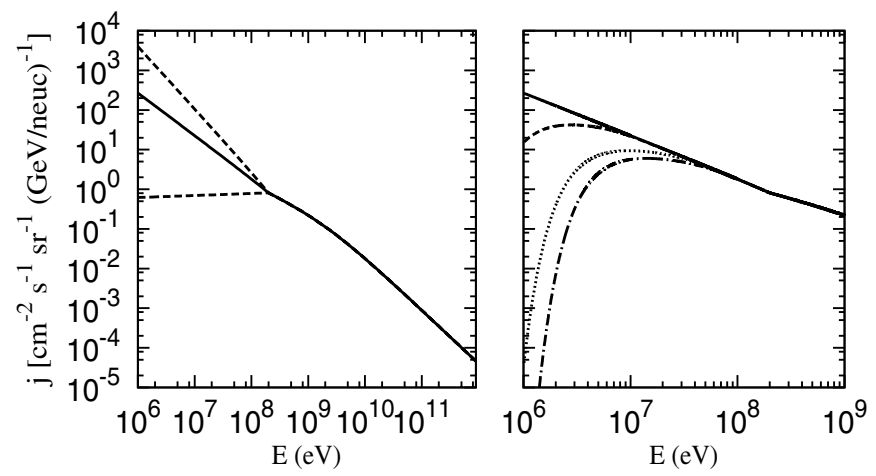

Figure 4. Flux spectrum of cosmic rays, $j(E)\left(\mathrm{cm}^{-2} \mathrm{~s}^{-1} \mathrm{sr}^{-1}(\mathrm{GeV}\right.$ nucleon $\left.{ }^{-1}\right)^{-1}$ ) vs. the cosmic ray energy, $E$, from Equation (17) (left), and as it varies with column density, $N_{\text {col }}$, according to our Monte Carlo cosmic ray propagation model (right). The left plot shows the effect of varying the parameter $\alpha$ that describes the power-law component of the spectrum below $2 \times 10^{8} \mathrm{eV}$. The solid line is for $\alpha=-2.15$, the dashed lines bound $-3.15<\alpha<0.1$. The right plot shows the flux spectrum with $\alpha=-2.15$ at $N_{\mathrm{col}}=0 \mathrm{~cm}^{-2}$ (solid), $1.5 \times 10^{21} \mathrm{~cm}^{-2}$ (dashed), $10^{22} \mathrm{~cm}^{-2}$ (dotted) and $2.5 \times 10^{22} \mathrm{~cm}^{-2}$ (dash-dot).

and (10)). A new spectrum is generated by binning the cosmic rays according to their energies, and then the cosmic rays are advanced another segment of the column, and the process is repeated.

At the very end of the process, we have a series of cosmic ray spectra, from the initial galactic cosmic ray spectrum at the edge of the exosphere, to the cosmic ray spectrum at the bottom of the cloud layer. The strength of Alfvén waves generated by cosmic rays depends on the difference between the initial cosmic ray spectrum and a given spectrum within the atmosphere.

Rimmer et al. (2012) consider the bulk of galactic cosmic rays to be positively charged (protons and alpha particles). This is the typical assumption, and is currently supported by observation (Webber 1998). These cosmic rays lose energy in the exosphere and atmosphere through ionizing collisions, until they are eventually thermalized. The result is a charge imbalance, with more positive charges present higher in the exosphere than in the exobase or upper atmosphere. This charge imbalance causes electrons to move from the exobase and upper atmosphere higher into the exosphere in order to neutralize the positive charge. These electrons will attempt to drag the magnetic field lines with them, generating Alfvén waves. The cosmic rays therefore lose energy in proportion to the energy of the Alfvén waves generated, in addition to the energy lost from collisions with the ambient gas. This mechanism for energy loss was first examined by Skilling \& Strong (1976), who considered cosmic ray exclusion in the interstellar medium, where magnetic fields are on the order of $3 \mu \mathrm{G}$. If the magnetic field is much stronger than this $(\gtrsim 1 \mathrm{mG})$, then the electrons will be more strongly locked to the magnetic field lines and they will no longer be able to efficiently generate Alfvén waves (i.e., the inequality in Equation (4) in Rimmer et al. 2012 will no longer be satisfied). Since this mechanism accelerates free electrons within the atmosphere, this may allow a cosmic ray driven current in atmospheric regions where the local magnetic field strength is $\leqslant 1 \mathrm{mG}$.

The question of the effect strong magnetic fields have on cosmic ray propagation is beyond the scope of this principle investigation. Large scale magnetic fields for brown dwarfs are several orders of magnitude greater than our $1 \mathrm{mG}$ limit (Reiners \& Basri 2007), so a study of strong magnetic field effects on cosmic ray transport is of great importance. Grießmeier 
et al. (2005) investigated cosmic ray propagation in exoplanet exospheres and atmospheres in the presence of Earth-strength magnetic fields, and found a correlation between the strength of the magnetic moment and anisotropy of cosmic rays on the surface of the planet (Grießmeier et al. 2005, their Figure 5). This anisotropy effect will increase for brown dwarfs as the strength of their magnetic field is suggested to be larger.

We take an initial flux density of cosmic rays, $j(E)\left(\mathrm{cm}^{-2} \mathrm{~s}^{-1}\right.$ $\left.\mathrm{sr}^{-1}\left(10^{9} \mathrm{eV} \text { nucleon }{ }^{-1}\right)^{-1}\right)$ to be the broken power-law spectrum from Indriolo et al. (2009), based on the models of Lerche $\&$ Schlickeiser (1982) and Shibata et al. (2006). This spectrum best fits both the observed light element abundances produced via scintillation as well as observed abundances of the ion $\mathrm{H}_{3}^{+}$ in the interstellar medium. The broken power-law spectrum changes sharply in flux at $<2 \times 10^{8} \mathrm{eV}$. This change results from "leaky-box" models (Lerche \& Schlickeiser 1982; Shibata et al. 2006), and is argued to be caused by low-energy shocks from either supernova remnants or possibly OB stellar atmospheres interacting with the ambient medium (Bykov \& Fleishman 1992). The initial flux density used in this paper is given as (based on Indriolo et al. 2009, their Equation (8)):

$$
j(E)= \begin{cases}j\left(E_{1}\right)\left(\frac{p(E)}{p\left(E_{1}\right)}\right)^{\gamma}, & \text { if } E>E_{2} \\ j\left(E_{1}\right)\left(\frac{p\left(E_{2}\right)}{p\left(E_{1}\right)}\right)^{\gamma}\left(\frac{p(E)}{p\left(E_{2}\right)}\right)^{\alpha}, & \text { if } E_{\text {cut }}<E<E_{2} \\ 0, & \text { if } E<E_{\text {cut }}\end{cases}
$$

where $p(E)=\frac{1}{c} \sqrt{E^{2}+2 E E_{0}}$ and $E_{0}=9.38 \times 10^{8} \mathrm{eV}$ is the proton rest energy. $E_{1}=10^{9} \mathrm{eV}$ and $E_{2}=2 \times 10^{8} \mathrm{eV}$ are constants, and the flux $j\left(E_{1}\right)=0.22 \mathrm{~cm}^{-2} \mathrm{~s}^{-1} \mathrm{sr}^{-1}$ $\left(10^{9} \mathrm{eV} \text { nucleon }^{-1}\right)^{-1}$ is the measured cosmic ray flux at $10^{9} \mathrm{eV}$. The fitting parameter $\gamma \approx-1.35$ is also well-constrained by observation (Mori 1997). The value for the second fitting exponent $\alpha$ depends on the models of Lerche \& Schlickeiser (1982) and Shibata et al. (2006); we choose the value $\alpha=-2.15$ because this value best agrees with the cosmic ray ionization in the interstellar medium inferred by Indriolo et al. (2007). The flux-spectrum in Equation (17) above $E \sim 10^{6} \mathrm{eV}$ is expected to be roughly the same throughout the interstellar medium (Strong \& Moskalenko 1998), and therefore seems to be a sensible initial cosmic ray flux applied at the outer edge of our exosphere. The parameter $\alpha$ determines the hardness of the LECR component of the spectrum, and has no observed lower limit, because LECRs are shielded from us by the solar wind. Upper limits to $\alpha$ can be determined from Voyager observations (e.g., Webber 1998). The effect on the spectrum from varying $\alpha$ can be seen in Figure 4. This figure also includes a plot showing how the cosmic ray spectrum changes with column density into our model atmospheres. An application of the results of Rimmer et al. (2012) to cosmic rays below $10^{6} \mathrm{eV}$ shows that cosmic rays are unlikely to travel farther than $\sim 1 \mathrm{pc}$ from their source of origin, the origin being either a supernova remnant or an $\mathrm{OB}$ association. It is therefore sensible to apply a low-energy cut-off, $E_{\text {cut }}=10^{6} \mathrm{eV}$, to the initial cosmic ray spectrum applied in this paper.

It is important to determine how the ionization rate changes when traveling into the exosphere and into the atmosphere. Fewer cosmic rays will be able to reach deeper into the atmosphere, so cosmic rays will affect the ambient gas less and less with increasing atmospheric depth. We now determine the cosmic ray ionization rate as a function of column density.
We apply Equation (17) to the Monte Carlo model from Rimmer et al. (2012) to determine a column-dependent flux density. This flux density, $j(E)$, can be used to calculate to the primary cosmic ray ionization rate for hydrogen, $\zeta_{p}\left(\mathrm{~s}^{-1}\right)$ by:

$$
\zeta_{p}=4 \pi\left(1+G_{10}\right) \int_{0}^{\infty}\left[1+\phi_{p}(E)\right] j(E) \sigma_{p}(E) d E .
$$

Here, $\sigma_{p}(E)$ is the ionization cross-section for a proton to ionize a hydrogen atom (Spitzer \& Tomasko 1968; Padovani et al. 2009) and $G_{10}$ is a factor representing the ionization by LECR electrons and by "heavy" LECRs (mostly $\alpha$-particles), and is assumed to be $G_{10}=0.8$ (Spitzer \& Tomasko 1968). The ionizing event produces a free electron at super-thermal energies, which often causes the ionization of additional species. This is accounted for in the term $\phi_{p}(E)$ which takes the form from Glassgold \& Langer (1973) and Padovani et al. (2009) of:

$$
\phi_{p}(E)=\frac{1}{\sigma_{p}(E)} \int_{I\left(\mathrm{H}_{2}\right)}^{\infty} P\left(E, E_{\mathrm{se}}\right) \sigma_{\mathrm{se}}\left(E_{\mathrm{se}}\right) d E_{\mathrm{se}}
$$

where $E_{\mathrm{se}}$ is the energy of the secondary electron, $I\left(\mathrm{H}_{2}\right)=$ $15.603 \mathrm{eV}$ is the ionization energy of molecular hydrogen, $\sigma_{e}$ $\left(\mathrm{cm}^{2}\right)$ is the cross-section for ionization of $\mathrm{H}_{2}$ by an electron (Mott 1930) and $P\left(E, E_{\mathrm{se}}\right)$ is the probability of the secondary electron having energy $E_{\mathrm{se}}$ given a proton of energy $E$. We approximate the Equation (19) as:

$$
\phi_{p}(E) \approx \frac{\sigma_{\mathrm{se}}\left(W(E)-I\left(\mathrm{H}_{2}\right)\right)}{\sigma_{p}(E)}
$$

where $W(E)$ is the average amount of energy deposited into the secondary electron from the cosmic ray proton, from Dalgarno et al. (1999). If $W(E)<I\left(\mathrm{H}_{2}\right)$ then $\phi_{p}=0$.

For HECRs, electrons are primarily produced by electronpositron production, muon decays, and other high-energy effects, and less so by ionizing collisions for which the crosssection is very small at high energies. Velinov et al. (2009) apply the CORSIKA code ${ }^{3}$ to realistic terrestrial atmospheric conditions (see Mishev \& Velinov 2008, 2010) in order to calculate the rate of electron production from HECRs, and find that it compares well with their analytical method (see also Velinov \& Mateev 2008). The HECR penetration is significantly affected by atmospheric composition (Molina-Cuberos et al. 1999). We therefore adapt the analytical method from Velinov \& Mateev (2008) to our atmosphere by making the following changes. Whereas Velinov \& Mateev (2008) set the ionization energy to that of nitrogen, $I\left(\mathrm{~N}_{2}\right)$, for the terrestrial atmosphere, we use an averaged ionization energy for the our atmospheric chemistry,

$$
I_{\mathrm{Av}} \approx \frac{I\left(\mathrm{H}_{2}\right) n\left(\mathrm{H}_{2}\right)+I(\mathrm{He}) n(\mathrm{He})+I(\mathrm{H}) n(\mathrm{H})}{n_{\mathrm{gas}}} .
$$

We apply the same methods to determine an average proton number, $Z_{\mathrm{Av}}$, and average mass number, $A_{\mathrm{Av}}$, for our atmospheric models. We also set the $E_{\min }=10^{9} \mathrm{eV}$ from Velinov \& Mateev (2008, their Equations (25) and (27)), because we treat LECR transport separately. The results of the analytic model of Velinov \& Mateev (2008), in terms of the electron production by HECRs, $Q_{\mathrm{HECR}}\left(N_{\mathrm{col}}\right)\left(\mathrm{cm}^{-3} \mathrm{~s}^{-1}\right)$, for giant gas planets and brown dwarf atmospheres, are shown in Figure 5. The production rate of

\footnotetext{
3 The CORSIKA code incorporates several models for hadron-hadron interactions as well as various parameterizations of Earth's atmosphere.
} 


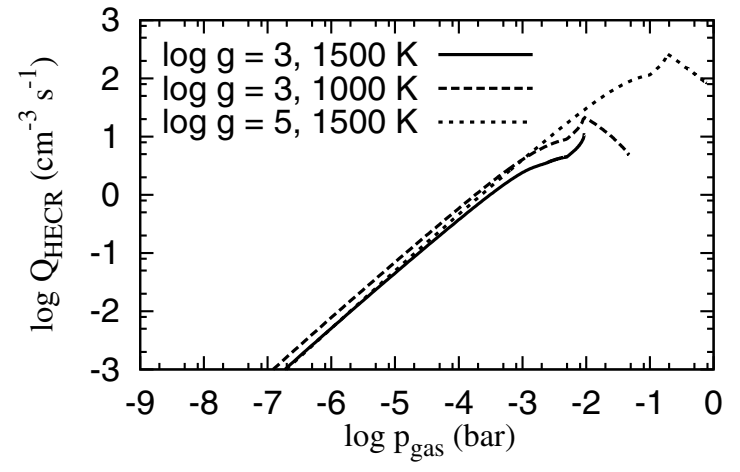

Figure 5. Electron production rate from cosmic rays of energies $E>10^{9} \mathrm{eV}$ $\left(Q_{\text {HECR }}\left(\mathrm{cm}^{-3} \mathrm{~s}^{-1}\right)\right)$ vs. pressure, $p_{\text {gas }}($ bar $)$, for both model giant gas planet atmospheres, with $\log g=3\left(T_{\text {eff }}=1500 \mathrm{~K}\right.$ (solid line) and $T_{\text {eff }}=1000 \mathrm{~K}$ (dashed line)) and brown dwarf atmosphere with $\log g=5, T_{\text {eff }}=1500 \mathrm{~K}$ (dashed line). The electron production rate, $Q_{\mathrm{HECR}}$, is obtained analytically from Velinov \& Mateev (2008). For the $\log g=3$ (giant gas planet) cases, the electron production peaks at $10^{-2}$ bar $(1000 \mathrm{~K})$ and 0.25 bar $(1500 \mathrm{~K})$. Electron production for the $\log g=5,1500 \mathrm{~K}$ (brown dwarf) case does not have a clear peak over the range of pressures we considered.

electrons depends on the gas column density according to Velinov \& Mateev (2008, their Equation (26) and (28)). The electron production for our $\log g=3$ model is within an order of magnitude of the electron production calculated for Jupiter's atmosphere from Whitten et al. (2008, their Figure 3(a)).

We combine the HECR results of Velinov \& Mateev (2008) and Velinov et al. (2009) to the Monte Carlo calculations for LECRs from Rimmer et al. (2012). This simply amounts to taking a total cosmic ray ionization rate, $Q\left(\mathrm{~cm}^{-3} \mathrm{~s}^{-1}\right)$ :

$$
Q\left(N_{\mathrm{col}}\right)=n_{\mathrm{gas}} \zeta_{\mathrm{LECR}}\left(N_{\mathrm{col}}\right)+Q_{\mathrm{HECR}}\left(N_{\mathrm{col}}\right)
$$

The column-dependent ionization rate as a function of column density, $N_{\text {col }}\left(\mathrm{cm}^{-2}\right)$, is well fit by the analytical form:

$$
\begin{aligned}
Q\left(N_{\mathrm{col}}\right)= & Q_{\mathrm{HECR}}\left(N_{\mathrm{col}}\right)+\zeta_{0} n_{\mathrm{gas}} \\
& \times \begin{cases}480 & \text { if } N_{\mathrm{col}}<N_{1} \\
1+\left(N_{0} / N_{\mathrm{col}}\right)^{1.2} & \text { if } N_{1}<N_{\mathrm{col}}<N_{2}, \\
e^{-k N_{\mathrm{col}}} & \text { if } N_{\mathrm{col}}<N_{2}\end{cases}
\end{aligned}
$$

where $\zeta_{0}=10^{-17} \mathrm{~s}^{-1}$ is the standard ionization rate in the dense interstellar medium, and the column densities $N_{0}=$ $7.85 \times 10^{21} \mathrm{~cm}^{-2}, N_{1}=4.6 \times 10^{19} \mathrm{~cm}^{-2}, N_{2}=5.0 \times 10^{23} \mathrm{~cm}^{-2}$, and $k=1.7 \times 10^{-26} \mathrm{~cm}^{2}$ are fitting parameters. Our calculated brown dwarf exospheric column density is within a factor of three of $N_{1}$, and the giant gas planet exospheric column density is about two orders of magnitude below $N_{1}$. In both cases, we do not expect the exosphere to have much impact on our cosmic ray transport model for giant gas planets and brown dwarfs. For atmospheric depths above $\approx 1.7 \mathrm{~g} \mathrm{~cm}^{-2}$, Equation (23) converges to Helling et al. (2012, their Equation (1)).

We calculate the degree of ionization using the same method as Whitten et al. (2008). We can estimate the steady-state number density of electrons, $n\left(e^{-}\right)\left(\mathrm{cm}^{-3}\right)$ using the rate equation:

$$
\frac{d n\left(e^{-}\right)}{d t}=Q-\alpha_{\mathrm{DR}}\left[n\left(e^{-}\right)\right]^{2},
$$

where $\alpha_{\mathrm{DR}}\left(\mathrm{cm}^{3} \mathrm{~s}^{-1}\right)$ is the recombination rate coefficient. This recombination rate coefficient includes both the coefficient for two-body recombination, $\alpha_{2}\left(\mathrm{~cm}^{3} \mathrm{~s}^{-1}\right)$, and three-body recombination, $\alpha_{3}\left(\mathrm{~cm}^{6} \mathrm{~s}^{-1}\right)$ such that:

$$
\alpha_{\mathrm{DR}}=\alpha_{2}+n_{\mathrm{gas}} \alpha_{3} .
$$

The two-body process is taken to be the recombination rate for protonated hydrogen (McCall et al. 2004, their Equation (7)),

$$
\alpha_{2} /\left(\mathrm{cm}^{3} \mathrm{~s}^{-1}\right)=8.22 \times 10^{-8}\left(\frac{T}{300 \mathrm{~K}}\right)^{-0.48}-1.3 \times 10^{-8}
$$

This equation agrees with the measured two-body recombination rate for protonated hydrogen over a temperature range of $10 \mathrm{~K}<T<4000 \mathrm{~K}$, and is in agreement with the typical two-body dissociative recombination rates for the upper atmosphere of Earth, according to Bardsley (1968). The three-body recombination rate is taken to be (Smith \& Church 1977):

$$
\alpha_{3} /\left(\mathrm{cm}^{6} \mathrm{~s}^{-1}\right)=2 \times 10^{-25}\left(\frac{T}{300 \mathrm{~K}}\right)^{-2.5} .
$$

We calculate the steady-state degree of ionization, $f_{e, \mathrm{CR}}=$ $n\left(e^{-}\right) / n_{\text {gas }}$, by setting $d n\left(e^{-}\right) / d t=0$ in Equation (24), and find:

$$
f_{e, \mathrm{CR}}=\frac{1}{n_{\mathrm{gas}}} \sqrt{\frac{Q}{\alpha_{\mathrm{DR}}}} .
$$

We will now combine this degree of ionization with the thermal degree of ionization and explore the resulting abundance of free electrons and their possible effect on the magnetic fields of these example objects.

\section{RESULTS AND DISCUSSION}

The total degree of ionization in the atmosphere can now be calculated by summing the degree of ionization, $f_{e, \mathrm{CR}}$ from Equation (28), discussed in Section 3, and the electron fraction due to thermal ionization included in the DrIFT-PhOENIX model atmosphere. The initial chemical abundances and the degree of thermal ionization, $f_{e \text {,thermal }}=n_{e \text {,thermal }} / n_{\mathrm{gas}}$, is provided by the Drift-Phoenix model with $T_{\text {eff }}=1500 \mathrm{~K}, \log g=3$ (giant gas planet), and $\log g=5$ (brown dwarf). The total degree of ionization, $f_{e}$, is then:

$$
f_{e}=f_{e, \text { thermal }}+f_{e, \mathrm{CR}} .
$$

Cosmic rays directly affect the number density of free electrons (Section 4.1). In Section 4.2 we analyze the effect of the free electron enhancement on the magnetic field coupling by evaluating the magnetic Reynolds number.

\subsection{Cosmic Ray Impact on the Electron Gas Density}

The impact of cosmic rays on the number of free electrons in the gas phase is the primary focus of our work. These results are plotted in Figures 6 and 7: for the $\log g=3$, $T_{\text {eff }}=1500 \mathrm{~K}$ giant gas planet the HECR component $\left(10^{9} \mathrm{eV}\right.$ $<E<10^{12} \mathrm{eV}$ ) dominates, and cosmic rays contribute substantially to the ionization until $p_{\text {gas }} \sim 10^{-3}$ bar. For the $\log g=3, T_{\text {eff }}=1000 \mathrm{~K}$ giant gas planet, the HECR component dominates until $10^{-2}$ bar. For the $\log g=5$ case (brown dwarf), the LECR component $\left(E<10^{9} \mathrm{eV}\right)$ dominates until $p_{\text {gas }} \sim 10^{-4}$ bar. The HECR component then takes over until $p_{\text {gas }} \sim 10^{-2}$ bar. Both the HECR and LECR components are 
Table 1

Effect of Cosmic Ray Ionization on Model Atmospheres by Region (See Figures 1, 6, and 7)

\begin{tabular}{|c|c|c|c|c|c|c|}
\hline $\begin{array}{l}g \\
\left(\mathrm{~cm} \mathrm{~s}^{-2}\right)\end{array}$ & $\begin{array}{l}T_{\text {eff }} \\
(\mathrm{K})\end{array}$ & $\begin{array}{c}p_{\text {gas }} \text { at the } \\
\text { Cloud Top } \\
\text { (bar) }\end{array}$ & $\begin{array}{c}p_{\text {gas }} \text { at the } \\
\text { Cloud Base } \\
\text { (bar) }\end{array}$ & $\begin{array}{c}p_{\text {gas }} \text { Where } \\
\text { HECR }^{\mathrm{a}} \\
\text { (bar) }\end{array}$ & $\begin{array}{c}p_{\text {gas }} \text { Where } \\
\text { LECR }^{\mathrm{b}} \\
\text { (bar) }\end{array}$ & $\begin{array}{c}p_{\text {gas }} \text { Where } \\
f_{e}>10^{-8} \\
\text { (bar) }\end{array}$ \\
\hline 3 & 1500 & $5 \times 10^{-5}$ & $10^{-2}$ & $10^{-7}-10^{-3}$ & $<10^{-7}$ & $<5 \times 10^{-10}$ \\
\hline 3 & 1000 & $2 \times 10^{-3}$ & $10^{-1}$ & $10^{-5}-5 \times 10^{-2}$ & $<10^{-5}$ & $<10^{-8}$ \\
\hline 5 & 1500 & $10^{-3}$ & $\sim 1$ & $10^{-6}-3 \times 10^{-2}$ & $<10^{-6}$ & $\ldots{ }^{c}$ \\
\hline
\end{tabular}

Notes.

a The region where cosmic rays of energy $10^{9} \mathrm{eV}<E<10^{12} \mathrm{eV}$ dominate.

b The region where cosmic rays of energy $E<10^{9} \mathrm{eV}$ dominate.

c $f_{e}<10^{-8}$ throughout this model atmosphere.
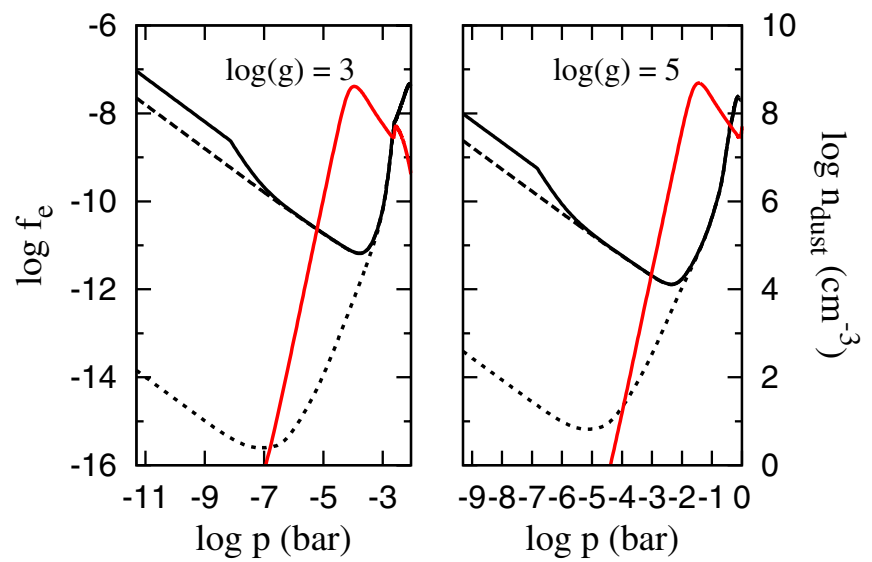

Figure 6. Degree of gas ionization, $f_{e}=n\left(e^{-}\right) / n_{\text {gas }}$, as a function of local gas pressure, for $T_{\text {eff }}=1500 \mathrm{~K}, \log g=3$ (giant gas planet, left), and $\log g=5$ (brown dwarf, right). The solid line denotes both the HECR and LECR contribution, while the dashed line denotes the HECR contribution only. The dotted line represents the electron abundance in the absence of cosmic rays, and demonstrates the extreme insufficiency of thermal ionization processes in cool objects. The solid red line is the dust number density, $n_{\text {dust }}\left(\mathrm{cm}^{-3}\right)$, and indicates where the cloud layer is located.

(A color version of this figure is available in the online journal.)

added to the local degree of thermal ionization that results from the DRIFT-PHOENIX model atmospheres. The regions where HECR and LECR components dominate for our three model atmospheres are given in Table 1.

Both the HECR and LECR components reach the cloud top, and the HECR component produces the bulk of free electrons within the upper $10 \%$ of cloud layer for the $\log g=3, T_{\text {eff }}=$ $1500 \mathrm{~K}$ (giant gas planet) atmosphere, $50 \%$ of cloud layer for the $\log g=3, T_{\text {eff }}=1000 \mathrm{~K}$ (giant gas planet) atmosphere and $5 \%$ of the cloud layer for the $\log g=5, T_{\text {eff }}=1500 \mathrm{~K}$ (brown dwarf) atmosphere. In regions where the LECR component dominates, the degree of ionization is enhanced by about a factor of five over what the HECR component would contribute alone. For the $\log g=3, T_{\text {eff }}=1000 \mathrm{~K}$ model atmosphere, the degree of ionization, $f_{e}$, exceeds $10^{-8}$ when $p_{\text {gas }}<10^{-8}$ bar. This approaches $f_{e} \sim 10^{-7}$, when the gas begins to act like a weakly ionized plasma (Diver 2001). The pressures below which this degree of ionization is reached are also included in Table 1. The brown dwarf atmosphere never achieves such a high degree of ionization. It is interesting to note in this context that clouds in the Earth atmosphere are not directly ionized by cosmic rays. Instead, the cosmic rays ionize the gas above the clouds and an ion current develops that leads to the ionization of the upper cloud layers (Nicoll \& Harrison 2010). Considering

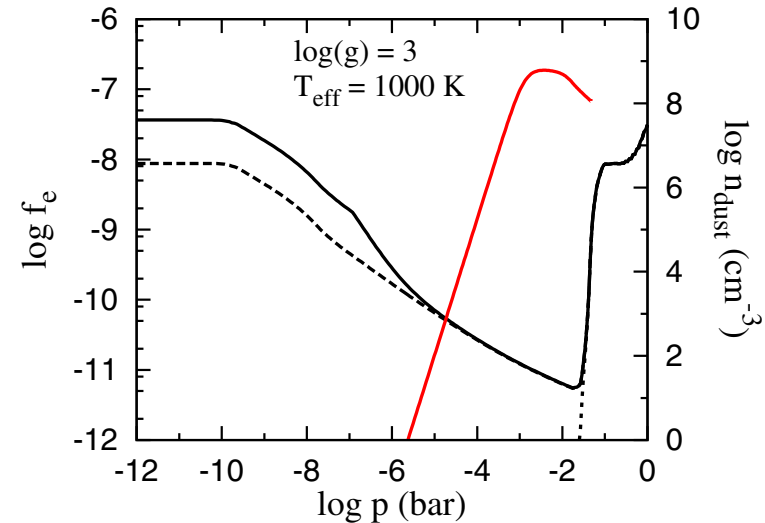

Figure 7. Degree of gas ionization, $f_{e}=n\left(e^{-}\right) / n_{\text {gas }}$, as a function of local gas pressure, for $\log g=3, T_{\text {eff }}=1000 \mathrm{~K}$. The solid line denotes both the HECR and LECR contribution, while the dashed line denotes the HECR contribution only. The dotted line represents the electron abundance in the absence of cosmic rays, hence thermal ionization only. The solid red line is the dust number density, $n_{\text {dust }}\left(\mathrm{cm}^{-3}\right)$, and indicates where the cloud layer is located.

(A color version of this figure is available in the online journal.)

how far cosmic rays penetrate into the cloud layers of our model atmospheres, it would be useful to explore the charging of grains by the resulting free electrons.

\subsection{Magnetic Field Coupling}

It is helpful to recast our results in terms of the degree the magnetic field couples to the gas. Helling et al. (2011b) quantify the degree of coupling by the magnetic Reynolds number, $R_{M}$, a dimensionless quantity which is directly proportional to the atmospheric degree of ionization, $f_{e}=p_{e} / p_{\mathrm{gas}}=n_{e} / n_{\mathrm{gas}}$, where $p_{e}$ and $p_{\text {gas }}$ are the electron pressure and gas pressure, respectively. The gas pressure and electron pressure are provided by the DRIFT-PHOENIX model atmospheres where the electron pressure is calculated from the Saha equation for thermal ionization processes.

The magnetic Reynolds number can be expressed as (Helling et al. 2011b):

$$
R_{M}=\left(10^{9} \mathrm{~cm}^{2} \mathrm{~s}^{-1}\right) \frac{4 \pi q^{2}}{m_{e} c^{2}} \frac{1}{\langle\sigma v\rangle_{\mathrm{en}}} f_{e},
$$

where $q$ is the electric charge, $m_{e}$ is the electron mass, $c$ is the speed of light, and $\langle\sigma v\rangle_{\text {en }}$ is the collisional rate, taken to be $\approx 10^{-9} \mathrm{~cm}^{3} \mathrm{~s}^{-1}$. The coupling of the magnetic field to the gas is expected if $R_{M}>1$, and though cosmic rays enhance $R_{M}$ in the outer atmosphere by orders of magnitude, the magnetic Reynolds number does not reach unity anywhere within the 


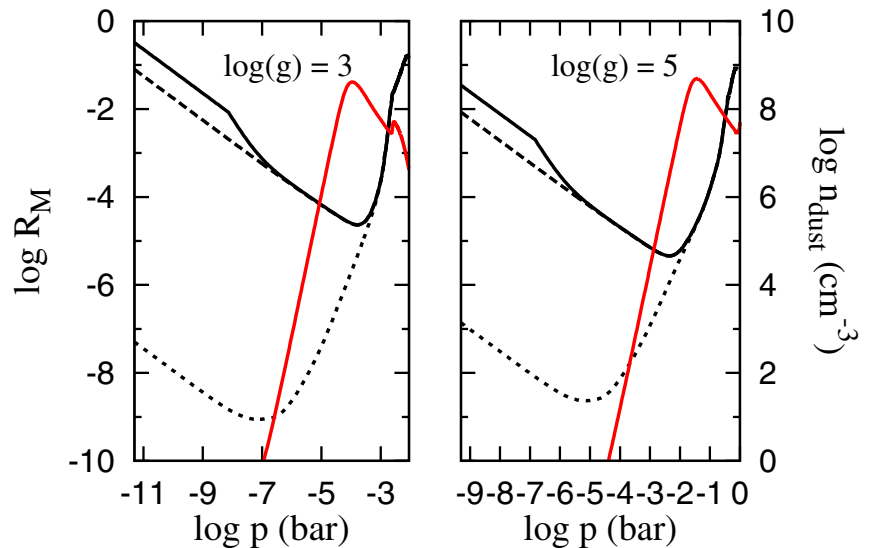

Figure 8. Magnetic Reynolds number (Equation (30)) for $T_{\text {eff }}=1500 \mathrm{~K}$, $\log g=3$ (giant gas planet, left) and $\log g=5$ (brown dwarf, right). The solid line denotes both the HECR and LECR contribution, while the dashed line denotes the HECR contribution only. The dotted line represents the electron abundance in the absence of cosmic rays. The solid red line is the dust number density, $n_{\text {dust }}\left(\mathrm{cm}^{-3}\right)$, and indicates where the cloud layer is located.

(A color version of this figure is available in the online journal.)

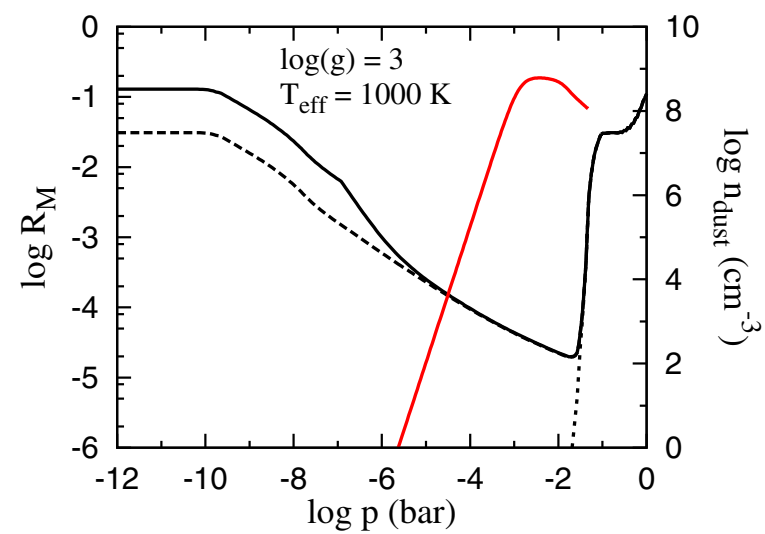

Figure 9. Magnetic Reynolds number (Equation (30)) for $\log g=3, T_{\text {eff }}=$ $1000 \mathrm{~K}$. The solid line denotes both the HECR and LECR contribution, while the dashed line denotes the HECR contribution only. The dotted line represents the electron abundance in the absence of cosmic rays, hence thermal ionization only. The solid red line is the dust number density, $n_{\text {dust }}\left(\mathrm{cm}^{-3}\right)$, and indicates where the cloud layer is located.

(A color version of this figure is available in the online journal.)

upper atmosphere (Figures 8 and 9). Since $R_{M}$ varies linearly with $f_{e}$, cosmic rays affect the magnetic Reynolds number over the same pressure range that they affect the degree of ionization for all model atmospheres. Since none of the model atmospheres achieves a value of $R_{M}>1$, this suggests that mechanisms other than cosmic rays of $E<10^{12} \mathrm{eV}$ will be needed if the magnetic field is to be coupled to the atmospheric gas in giant gas planets or brown dwarfs.

\section{SUMMARY}

This paper seeks to answer the question of the significance of cosmic ray ionization on the number of free electrons in brown dwarfs and giant gas planets. We further examine the possible coupling of the magnetic field to the gas because of the increased degree of ionization. We develop an analytical model for the exospheric density profile which we combine to an atmospheric structure. The DRIFT-PHOENIX model atmospheres provide the inner boundary conditions for the exosphere model, and they provide the density profile that we utilize below the exobase. Cosmic ray transport through the exosphere and the atmosphere is then calculated using these density profiles. A Monte Carlo cosmic ray transport method from Rimmer et al. (2012) is applied to cosmic rays of $E<10^{9} \mathrm{eV}$. An analytic method for cosmic ray transport from Velinov \& Mateev (2008) is applied to cosmic rays of $E>10^{9} \mathrm{eV}$. We calculate an ionization rate for which we provide a parameterized expression (Equation (23)). We use this expression to estimate the steadystate degree of ionization (Equation (29)).

Do cosmic rays have a significant impact on the electron fraction? If the measure of significance is the number of free electrons in the upper atmosphere, then the answer is "yes." Cosmic ray ionization is responsible for almost all the free electrons in our upper model atmospheres and for the giant gas planet model atmospheres, achieves a degree of ionization approaching that necessary to qualify the highest regions of these model atmospheres as weakly interacting plasmas, thereby providing an environment for plasma processes (Stark et al. 2013). If, however, the measure of significance is the degree of coupling of the magnetic field to the gas, then the answer is "no." The model predicts a cosmic ray enhancement to the steadystate electron abundance by several orders of magnitude for atmospheric regions with $p_{\text {gas }}<10^{-3}$ bar for brown dwarf conditions and for $p_{\text {gas }}<10^{-2}, 10^{-4}$ bar for giant gas planet conditions, with $T_{\text {eff }}=1500 \mathrm{~K}$ in both cases. This enhancement is not large enough to allow the magnetic Reynolds number, $R_{M}>1$ above the cloud top, and does not significantly affect $R_{M}$ for the bulk of the cloud layer. This indicates that the magnetic field would not couple to the gas because of the steady-state cosmic ray ionization enhancement. However, the geometry of the magnetic field (e.g., Donati et al. 2008; Vidotto et al. 2012; Lang et al. 2012) might lead to a channeling of the cosmic rays which then would amplify the local degree of ionization beyond the values determined in this paper, which would cause heterogeneous distribution of cosmic ray induced chemical products. The increased abundance of electrons may contribute to charge build-up on dust at the top of the cloud layer. This is especially the case for our model brown dwarf atmosphere, because cosmic rays penetrate more deeply into its cloud layer.

Cosmic rays were first discovered through their ionization effects in the Earth's atmosphere. We predict that ionization effects have a significant impact on the upper atmospheres of free-floating extrasolar planets and very low-mass stars. The effect the cosmic ray induced degree of ionization has on the chemistry in the upper atmospheres of these objects is a very interesting question. It has been suggested that cosmic rays may drive production of small hydrocarbons that may be responsible for the hazes of, e.g., HD 189733 b (Moses et al. 2011). This is a question we hope to explore in detail in a future paper.

We highlight financial support of the European Community under the FP7 by an ERC starting grant. We are grateful to Kristina Kislyakova and Helmut Lammer for helpful discussions relating to our exosphere model.

\section{REFERENCES}

Bardsley, J. N. 1968, JPhB, 1, 365

Bazilevskaya, G. A., Usoskin, I. G., Flückiger, E. O., et al. 2008, SSRv, 137, 149

Berger, E., Basri, G., Fleming, T. A., et al. 2010, ApJ, 709, 332

Burrows, A., Hubbard, W. B., Lunine, J. I., \& Liebert, J. 2001, RvMP, 73, 719

Bykov, A. M., \& Fleishman, G. D. 1992, SvAL, 18, 95

Cesarsky, C. J., \& Volk, H. J. 1978, A\&A, 70, 367 
Chabrier, G., Baraffe, I., Allard, F., \& Hauschildt, P. 2000, ApJ, 542, 464

Chamberlain, J. W., \& Hunten, D. M. 1987, Theory of Planetary Atmospheres. An Introduction to Their Physics and Chemistry (International Geophysics Series, Vol. 36; Orlando, FL: Academic)

Cravens, T. E., \& Dalgarno, A. 1978, ApJ, 219, 750

Dalgarno, A., Yan, M., \& Liu, W. 1999, ApJS, 125, 237

Dehn, M. 2007, PhD thesis, Univ. Hamburg

Diver, D. A. 2001, A Plasma Formulary for Physics, Technology, and Astrophysics (Berlin: Wiley-VCH)

Donati, J.-F., Morin, J., Petit, P., et al. 2008, MNRAS, 390, 545

Ermakov, V. I., \& Stozhkov, Y. 2003, in Solar Variability as an Input to the Earth's Environment, ed. A. Wilson (Noordwijk: ESA), 359

Galli, A., Wurz, P., Lammer, H., et al. 2006, SSRv, 126, 447

Glassgold, A. E., \& Langer, W. D. 1973, ApJ, 186, 859

Grießmeier, J.-M., Stadelmann, A., Motschmann, U., et al. 2005, AsBio, 5, 587

Helling, C., Ackerman, A., Allard, F., et al. 2008, MNRAS, 391, 1854

Helling, C., Jardine, M., Diver, D., \& Witte, S. 2012, P\&SS, 77, 152

Helling, C., Jardine, M., \& Mokler, F. 2011a, ApJ, 737, 38

Helling, C., Jardine, M., Witte, S., \& Diver, D. A. 2011b, ApJ, 727, 4

Hess, V. F. 1912, PhyZ, 13, 1084

Hinteregger, H. E., Fukui, K., \& Gilson, B. R. 1981, GeoRL, 8, 1147

Indriolo, N., Fields, B. D., \& McCall, B. J. 2009, ApJ, 694, 257

Indriolo, N., Geballe, T. R., Oka, T., \& McCall, B. J. 2007, ApJ, 671, 1736

Jokipii, J. R. 1976, Nablyud. i Prognoz Soln. Aktivnosti (Moskva: Mir), 213

Lammer, H., Selsis, F., Ribas, I., et al. 2003, ApJL, 598, L121

Lang, P., Jardine, M., Donati, J.-F., Morin, J., \& Vidotto, A. 2012, MNRAS, 424, 1077

Lerche, I., \& Schlickeiser, R. 1982, MNRAS, 201, 1041

Marois, C., Macintosh, B., Barman, T., et al. 2008, Sci, 322, 1348

McCall, B. J., Huneycutt, A. J., Saykally, R. J., et al. 2004, PhRvA, 70, 052716

Mishev, A. L., \& Velinov, P. I. Y. 2008, CRABS , 61, 639

Mishev, A. L., \& Velinov, P. I. Y. 2010, JASTP, 72, 476

Molina-Cuberos, G. J., López-Moreno, J. J., Rodrigo, R., \& Lara, L. M. 1999, JGR, 104, 21997

Mori, M. 1997, ApJ, 478, 225
Moses, J. I., Visscher, C., Fortney, J. J., et al. 2011, ApJ, 737, 15

Mott, N. F. 1930, RSPSA, 126, 259

Muraki, Y., Axford, W. I., Matsubara, Y., et al. 2004, PhRvD, 69, 123010

Murray-Clay, R. A., Chiang, E. I., \& Murray, N. 2009, ApJ, 693, 23

Nicoll, K. A., \& Harrison, R. G. 2010, GeoRL, 37, 13802

Padovani, M., Galli, D., \& Glassgold, A. E. 2009, A\&A, 501, 619

Pierrard, V., \& Lemaire, J. 1996, JGR, 101, 7923

Pudovkin, M. I., \& Veretenenko, S. V. 1995, JATP, 57, 1349

Reiners, A., \& Basri, G. 2007, ApJ, 656, 1121

Rimmer, P. B., Herbst, E., Morata, O., \& Roueff, E. 2012, A\&A, 537, A7

Rudd, M. E., Goffe, T. V., Dubois, R. D., Toburen, L. H., \& Ratcliffe, C. A. 1983, PhRvA, 28, 3244

Shibata, T., Hareyama, M., Nakazawa, M., \& Saito, C. 2006, ApJ, 642, 882

Shumilov, O. I., Kasatkina, E. A., Henriksen, K., \& Vashenyuk, E. V. 1996, AnG, 14, 1119

Skilling, J., \& Strong, A. W. 1976, A\&A, 53, 253

Smith, D., \& Church, M. J. 1977, P\&SS, 25, 433

Spitzer, L. 1978, Physical Processes in the Interstellar Medium (New York: Wiley)

Spitzer, L., Jr., \& Tomasko, M. G. 1968, ApJ, 152, 971

Stark, C. R., Helling, Ch., Diver, D. A., \& Rimmer, P. B. 2013, ApJ, submitted Stozhkov, Y. I. 2003, JPhG, 29, 913

Strong, A. W., \& Moskalenko, I. V. 1998, ApJ, 509, 212

Umebayashi, T., \& Nakano, T. 1981, PASJ, 33, 617

Velinov, P. I. Y., \& Mateev, L. N. 2008, JASTP, 70, 574

Velinov, P. I. Y., Mishev, A., \& Mateev, L. 2009, AdSpR, 44, 1002

Vidal-Madjar, A., Désert, J.-M., Lecavelier des Etangs, A., et al. 2004, ApJL, 604, L69

Vidotto, A. A., Fares, R., Jardine, M., et al. 2012, MNRAS, 423, 3285

Watson, A. J., Donahue, T. M., \& Walker, J. C. G. 1981, Icar, 48, 150

Webber, W. R. 1998, ApJ, 506, 329

Webber, W. R., \& Higbie, P. R. 2009, JGRA, 114, 2103

Whitten, R. C., Borucki, W. J., O'Brien, K., \& Tripathi, S. N. 2008, JGRE, 113,4001

Witte, S., Helling, C., \& Hauschildt, P. H. 2009, A\&A, 506, 1367 